\title{
EINE EIGENSCHAFT DER PRIMZAHL 107
}

$$
\text { TON }
$$

\section{K. SCHWERING}

in COESFELD.

In meinem Aufsatze Über gewisse trinomische komplexe Zahlen ${ }^{1}$ habe ich auf die Primzahl ro7 als für eine Frage der Zahlentheorie szur Untersuchung einladends hingewiesen. Ich bin nunmehr in der Lage, die dort (Seite 84, Fussnote) gemachten Mitteilungen zu vervollständigen und mit Hülfe des ganzen Ergebnisses die Frage:

》Ist es immer möglich, die von $\mathrm{J}_{\text {ACOBI }}$ mit $(\alpha, x)^{\lambda}$ bezeichneten Kreisteilungsausdrücke als Produkte konjugirter $\phi$-Funktionen darzustellen?D durch ein zweites Beispiel in verneinendem Sinne zu beantworten.

Nimmt man als primitive Wurzel 2, so erhalten wir die folgenden 18 Zahlen:

ferner:

$$
\mathrm{I}+\alpha+\alpha^{r} \text { für } r=4,3 \mathrm{I}, 47,50
$$

$$
\begin{aligned}
1+\alpha-\alpha^{r} \text { für } r= & 43,50,35,30,32,17,17,38, \\
& 41,7,51,47,22 ;
\end{aligned}
$$

endlich:

$$
2+a
$$

deren Normen unter der Voraussetzung $\alpha^{53}=\mathrm{I}$ zu bilden sind. Zunächst tritt $r=17$ zweimal auf; dann ist nach Formel 25 obiger Abhandlung:

$$
\begin{aligned}
N\left(\mathrm{I}+\alpha+\alpha^{30}\right)= & N\left(\mathrm{I}+\alpha+\alpha^{4}\right), \quad N\left(\mathrm{I}+\alpha-\alpha^{47}\right)=N\left(\mathrm{I}+\alpha-\alpha^{3}\right), \\
& N\left(\mathrm{I}+\alpha-\alpha^{22}\right)=N\left(\mathrm{I}+\alpha-\alpha^{32}\right) .
\end{aligned}
$$

1 Diese Zeitschrift, Bd. IO, S. $57 \mathrm{ff}$.

Acta nathematica. 11. Imprimé le 19 Janpier 1888. 
Für die übrigen erhält man:

$$
\begin{array}{ll}
N\left(\mathrm{1}+\alpha+\alpha^{4}\right)=107.181579, & N\left(\mathrm{1}+\alpha+\alpha^{31}\right)=107.76003, \\
N\left(1+\alpha+\alpha^{41}\right)=107.118297, & N\left(1+\alpha-\alpha^{43}\right)=107.151051, \\
N\left(1+\alpha-\alpha^{50}\right)=107.243589, & N\left(1+\alpha-\alpha^{35}\right)=107.246769, \\
N\left(1+\alpha-\alpha^{30}\right)=107.73459, & N\left(1+\alpha-\alpha^{32}\right)=107.107 .7103, \\
N\left(1+\alpha-\alpha^{17}\right)=107.107 .1061, & N\left(1+\alpha-\alpha^{38}\right)=107.191119 ., \\
N\left(1+\alpha-\alpha^{4}\right)=107.27773, & N\left(1+\alpha-\alpha^{i}\right)=107.107 .3181, \\
N\left(1+\alpha-\alpha^{51}\right)=107.27773 . &
\end{array}
$$

Endlich

$$
N(2+x) \cdot=3.107 .28059810762433 .
$$

Die Gleichheit der Normen $N\left(1+\alpha-\alpha^{41}\right)=N\left(1+\alpha-\alpha^{51}\right)$ ist bemerkenswert, da die Zahlen $4 \mathrm{I}$ und $5 \mathrm{I}$ verschiedenen Gruppen (obige Abhandl. S. 69) nămlich $\mu=4$ und $\mu=2$ angehören.

In der oben erwähnten Fussnote heisst es durch einen Druckfehler I $+\alpha-\alpha^{4}$ statt I $+\alpha+\alpha^{4}$, wie oben angegeben.

Ich benutze die Gelegenheit zu der ferneren Mitteilung uber die Normen $N\left(z+\alpha-\alpha^{n+1}\right)$, wo $\alpha^{\lambda}=\mathrm{I}, \lambda$ reelle Primzahl und $\mu$ eine beliebige ganze Zahl ist. In den zahlreichen von mir berechneten Beispielen war der Koefficient jeder geraden Potenz von $z$, also der Koefficient von $z^{2}, z^{4}, z^{6}, \mathrm{u}$. s. w. stets entweder Null oder eine positive Zahl.

Im September I 887 . 